\title{
CASE REPORT FORM
}

\section{Pt ID\#}

\section{Clinical history:}

General appearance:

Infection site:
Week 0: Date:

Signs and Symptoms: $\quad$ Duration of symptoms since:

\begin{tabular}{|c|c|c|c|c|c|c|}
\hline & $\underline{\text { Yes }}$ & $\underline{\text { No }}$ & & $\underline{\text { Yes }}$ & $\underline{\text { No }}$ & \\
\hline 1. Fever & $\square$ & $\square$ & 5. Contact w/active case & $\square$ & $\square$ & 9. Weight $(\mathrm{kg})_{-}$ \\
\hline 2. Cough & $\square$ & $\square$ & 6. Loss of appetite & $\square$ & $\square$ & 10. Others (Specify) \\
\hline 3. Chest pain & $\square$ & $\square$ & 7. Nausea & $\square$ & $\square$ & \\
\hline 4. Haemoptysis & $\square$ & $\square$ & 8. Anorexia & $\square$ & $\square$ & \\
\hline
\end{tabular}

Other information: $\quad \underline{\text { Yes }} \quad \underline{\text { No }} \quad \underline{\text { Yes }} \quad \underline{\text { No }} \quad \underline{\text { Yes }}$ 1. Previous H/O TB $\square \quad \square \quad$ 2. BCG given $\square \quad \square \quad$ 3. Current steroid use $\square \quad \square$

Laboratory:

1. $\mathrm{AFB}$ (score, $0-3+) \quad \square 0 \quad \square 1+\quad \square 2+\quad \square 3+$

2. L-J culture $\square$ neg $\square$ pos $\quad$ \#colonies $\square$ 1+ $\square$ 2+ $\square$ 3+ $\square$ 4+ $\square$ conta/lost

3. Sensitivity: Rif INH ETH PZA STR

4. PPD $\square$ prior positive $\square$ current $\square \mathrm{mm} \quad \square$ not done

5. Chest X-ray $\%$ involvement

6. Blood: ESR $\mathrm{Hb}$ TC Neutrophils Lymphocytes

\section{Week 1: Follow up date:}

1. a) Received anti-TB treatment $\square$ regular $\quad \square$ irregular

b) Received supplement

$\square$ regular $\square$ irregular

2. Fever:

5. Appetite:

7. Joint pain:

3. Cough:

6. Anorexia:

8. Jaundice:

4. Weight $(\mathrm{kg})$ :

9. AFB (score, $0-3+$ )

$\square$

$\square 1+$

$\square^{2+} \square 3+$

no sputum

Comments: 


\section{Week 2: Follow up date:}

1. a) Received anti-TB treatment

$\square$ regular $\square$ irregular

b) Received supplement

$\square$ regular

$\square$ irregular

2. Fever:

5. Appetite:

3. Cough:

6. Anorexia:

7. Joint pain:

4. Weight $(\mathrm{kg})$ :

9. AFB (score, $0-3+$ ):

$\square 0 \quad \square 1+\square 2+\square 3+\square$ no sputum

Comments:

\section{Week 3: Follow up date:}

1. a) Received anti-TB treatment $\square$ regular $\square$ irregular

b) Received supplement $\quad \square$ regular $\square$ irregular

2. Fever:

5. Appetite:

7. Joint pain:

3. Cough:

6. Anorexia:

8. Jaundice:

4. Weight $(\mathrm{kg})$ :

9. AFB (score, $0-3+)$ :

$\square 0 \quad \square 1+\square 2+\square 3+\square$ no sputum

Comments:

\section{Week 4: Follow up date:}

1. a) Received anti-TB treatment

$\square$ regular $\square$ irregular

b) Received supplement

$\square$ regular $\quad \square$ irregular

2. Fever:

5. Appetite:

7. Joint pain:

3. Cough:

6. Anorexia:

8. Jaundice:

4. Weight $(\mathrm{kg})$ :

9. $\mathrm{AFB}$ (score, $0-3+$ ):

$\square 0 \quad \square 1+\square 2+\square 3+\square$ no sputum

10. L-J culture: $\square$ neg $\square$ pos (\#colonies $\square 1+\quad \square 2+\quad \square 3+\square 4+\quad \square$ conta/lost

12. Blood: ESR $\mathrm{Hb}$ $\mathrm{TC}$ Neutrophils Lymphocytes

Comments:

Week 6: Follow up date:

1. a) Received anti-TB treatment

$\square$ regular $\square$ irregular

b) Received supplement

$\square$ regular $\square$ irregular

2. Fever:

5. Appetite:

7. Joint pain:

3. Cough:

6. Anorexia:

8. Jaundice:

4. Weight $(\mathrm{kg})$ :

9. AFB (score, $0-3+$ ):

$\square 0 \quad \square 1+\quad \square 2+\square 3+\square$ no sputum 
Comments:

\section{Week 8: Follow up date:}

1. a) Received anti-TB treatment

$\square$ regular $\square$ irregular

b) Received supplement

$\square$ regular $\quad \square$ irregular

2. Fever:

5. Appetite:

7. Joint pain:

3. Cough:

6. Anorexia:

8. Jaundice:

4. Weight $(\mathrm{kg})$ :

9. AFB (score, $0-3+$ ): $\quad \square 0 \quad \square 1+\square 2+\square 3+\square$ no sputum

10. L-J culture: $\square$ neg $\square$ pos (\#colonies $\square 1+\quad \square 2+\quad \square 3+\square 4+\quad \square$ conta/lost

11. Chest X-ray $\%$ involvement

12. Blood: ESR $\mathrm{Hb}$ TC Neutrophils Lymphocytes

Comments:

\section{Week 10: Follow up date:}

1. Received anti-TB treatment $\square$ regular $\square$ irregular

2. Fever:

5. Appetite:

6. Anorexia:

3. Cough:

4. Weight $(\mathrm{kg})$ :

9. AFB (score, 0-3+):

Comments:

\section{Week 12: Follow up date:}

1. Received anti-TB treatment $\square$ regular

2. Fever:

5. Appetite:

7. Joint pain:

3. Cough:

6. Anorexia:

8. Jaundice:

4. Weight (kg):

9. $\mathrm{AFB}$ (score, $0-3+)$ :

10. Chest X-ray

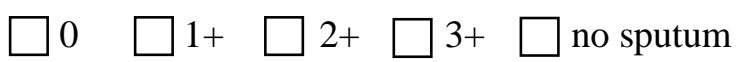
$\%$ involvement
7. Joint pain:

8. Jaundice:

Comments:

\section{Week 24: Follow up date:}

1. Received anti-TB treatment $\square$ regular $\square$ irregular

2. Fever:

5. Appetite:

6. Anorexia:

3. Cough:

4. Weight $(\mathrm{kg})$ :

9. AFB (score, $0-3+)$ : 
10. Chest X-ray $\%$ involvement

Comments: 